\title{
USING HYBRID SIMULATION TO REPRESENT THE HUMAN FACTOR IN PRODUCTION SYSTEMS
}

\author{
Oliveira, M. L. M.; Montevechi, J. A. B.; Pinho, A. F. \& Miranda, R. C. \\ Federal University of Itajuba (UNIFEI), \\ Av. BPS, 1303, Pinheirinho Itajuba, MG, ZIP CODE: 37500-000, Brazil \\ E-Mail: monaoli@yahoo.com.br, montevechi@unifei.edu.br, pinho@unifei.edu.br, \\ mirandaprod@yahoo.com.br
}

\begin{abstract}
Discrete-event simulation (DES) has been widely used in systems analysis. However, authors defend the idea that this kind of simulation does not properly represent the human element or the factors affecting its productivity. In Agent-Based Simulation (ABS), agents are intelligent, autonomous and proactive beings, which are features closely related to human behaviour. Therefore, ABS and DES were combined into one model to represent the human element as agents, who were subjected to one of the factors that affect their performance, the circadian rhythm. After statistical validation it was determined that the computer models integrating ABS and DES represent the productive systems under analysis with high level of confidence. A comparison between the model built and a DES model where operators are represented by resources was also performed. It was verified that the ABS/DES represents better the actual systems behaviour than the DES, showing a lower average of relative error. (Received in August 2016, accepted in December 2016. This paper was with the authors 1 month for 1 revision.)
\end{abstract}

Key Words: Agent-Based Simulation, Circadian Rhythm, Discrete-Event Simulation

\section{INTRODUCTION}

Computer simulation has become a well-known and often used tool by companies in an attempt to analyse and optimize their processes. Actual production systems are dynamic and random, and simulation projects seek to represent these characteristics. Computer simulation can be defined as the process of developing an actual or hypothetical system model in order to conduct experiments to understand the behaviour of this system [1]. Simulation modelling is the activity of reducing complexity of the real world [2].

Manufacturing systems are composed of many discrete operations that occur random and non-linearly, often causing mathematical models or other methods to be impractical [3]. This is one reason why many companies use discrete-event simulation to learn about and explore scenarios of their processes. In discrete-event simulation, the modeller considers the system being modelled as a process, i.e., a sequence of operations being performed through entities [4].

However, there are situations where the use of discrete-event simulation alone may not be effective, for example, in situations where the representation in greater detail of the human element in simulation projects is required. In these cases, the modeller must make very accurate assumptions about the decisions of the human element in order to adjust their behaviour in the modelling format of discrete-event simulation. Real-time decisions of individual entities would be very difficult to model using this type of simulation [5].

Computer simulation often overestimates the production capacity of manufacturing systems and this difference between the expected and the actual performance is due to the fact that simulation models do not incorporate key elements such as human performance and the factors impacting this performance. Most simulation software represents in many details the machine behaviour, but considers workers as mere resources [6, 7].

Agent-based simulation is different from the classical discrete-event simulation because of the nature of the agents, in Agent-Based Simulation (ABS) they are proactive, independent 
and intelligent [8]. In many studies in the literature, researchers have used agent-based simulation to represent the human element, since these characteristics of agents are strongly related to characteristics of the human being. ABS helps in a better understanding of realworld systems where the representation or modelling of people is important and individuals have autonomous behaviours [9].

By combining discrete-event simulation and agent-based simulation, it is possible to model aspects of the system that could not be simulated by any of the simulation methods separately [5]. Is it possible to benefit from the combination of discrete-event with agentbased simulation to represent the human element and the factors affecting its performance in order to make the simulation result closer to reality?

To address this question, this paper aims to report the results of an agent-based simulation model combined with discrete-event simulation of different production processes, where an agent represents each employee. These agents have decision-making autonomy and are susceptible to one of the factors that affect human performance, the circadian rhythm. Productivity results from these computer models are compared with the actual system through statistical test to verify that it represents the behaviour of the cell in question. Besides, these agent-based simulation models were compared with discrete-event simulation models to determine which one is closer to the reality.

This paper is organized as follows: theoretical foundation, where the main references on the subject are presented; research method adopted; application of this method; analysis of the main results and conclusion.

\section{BACKGROUND}

\subsection{Discrete-event simulation}

Simulation is a problem solving methodology necessary for solving many real-world problems, since it can be used to describe and analyse the behaviour of a system [10]. Computer simulation can be defined as the virtual representation of a real life system through a model, making it possible to study this model without having to actually build it or even make changes in the system, and study the results of these changes without the need to change it in advance [11].

Exploring scenarios in a virtual environment without physical risks and/or high costs is one of the great advantages of simulation projects. It does not solve by itself the problems related to the system, but provides results and outputs that can be used in solving these problems [12].

However, there are some situations which are difficult to model using only DES, for example in situations where the modeller must make very accurate assumptions about the decisions of the human element, in order to adjust their behaviour in the modelling format of discrete-event simulation [5]. Some authors support the idea that not properly representing the human factor can impair the results of computer models. We need to represent people realistically considering their behaviour and performance in order to improve the accuracy of the simulation [13].

\subsection{Agent-based simulation}

Agent-Based Simulation can be considered a new modelling paradigm for the next generation of manufacturing systems design; it allows inserting a high degree of individualization, autonomy and interactivity to the agents considered in the modelled system [14]. ABS differs from the classical discrete-event simulation (DES) due to the nature of agents, once ABS agents are proactive, independent and intelligent, can initiate actions, communicate with other 
agents and make decisions on their own. In DES, entities are simple, reactive and with limited capacity [8].

Agent-based simulation has been used to model different situations over the past decades, such as social development, segregation, spread of diseases, effectiveness of advertisements and in simulation of industries as well [5]. Moreover, ABS has been applied in several fields such as e-commerce, business, air traffic control, process control, telecommunications, and manufacturing applications [15].

The agent based simulation is used for testing new ideas, to predict the effect of a new technology, developing new theory, to aid decision-making and what-if training, conducting critical experiments and hypotheses generators [16]. The main software for this type of simulation are Swarm $\AA$, Repast $\AA$, NetLogo $\AA$, AnyLogic $\AA$, MASON $\AA$ and Ascape $\AA$. However, the most advanced simulator is Anylogic ${ }^{\circledR}$ commercial software, which allows modelling in an integrated way all the main simulation paradigms [17].

\subsection{The human factor in simulation projects}

There is a recent necessity of observing, analysing and predicting human behaviour through the use of computer simulation technology [18]. Despite the various numbers of studies recognizing the importance of human performance, many of them concentrate inappropriately in the technical performance instead of the human components performance involved in the system [19].

Discrete Event Simulation is one of the most powerful tools for planning, designing and improving material movements in production [20]. Discrete Event Simulation models play an important role in the improvement of the production cells efficiency; however, most of the simulation projects consider the human being as a common production resource, ignoring the influence of the organization behaviour in the production process [21]. The results predicted using DES models are different from the results that occur in a real life due to the incapacity of a simulation model to represent precisely the workers performance [22]. To amplify this modelling capacity, especially in the simulation using discrete events, it is important to evaluate how the human factors can impact the production system [23].

The ABS has the potential to become the predominant paradigm to model the human behaviour [17]. The agent based simulation is a powerful and flexible tool to model complex systems made up by several components that interacts with each other, like the ones that involves humans and their behaviour [24, 25].

In order to comprehend how human elements has been represented in works that use DES and ABS projects, a systematic review was made with articles that used as keywords: "Agent Based Simulation", "Discrete Event Simulation" and keywords related to the human factor such as "Human Performance", "Human Element", among others. The articles that used DES and had keywords related to human factor sought to represent the human element in simulation through the variation of human performance. In these articles, the performance was affected by the alteration of cognitive elements, the calculation of the probability of an error occurring, but in the great majority of cases, by lead time variation of activities measured through timing analysis.

For the papers that used ABS and had keywords related to the human factor, it was possible to verify that they were related to the representation of behaviour in different situation and these behaviour were the result of personal and cognitive characteristics, actions and even emergency situations as display alerts and evacuation. However it is important to observe that none of these papers were related to the industrial sector, not seeking to comprehend the human performance in the manufactory environment, reinforcing the importance of this article. 


\subsection{Circadian rhythm}

Operator can be integrated into a simulation model as an agent or as one of its components [26]. In this paper, it is necessary to assign to agents behavioural factors that affect their performance throughout the day to better represent the human element in the computational model developed. When selecting models that represent the behaviour of human performance, the following criteria must be considered: the model should be valid, have enough literature to indicate that the factor represented could be present in the manufacturing context and the input data are easily obtained [6]. Therefore, based on these criteria, the equation of [27] was chosen to represent the influence of the circadian rhythm in human performance.

Human beings are creatures that are susceptible to a drop in performance and an increase of sleepiness in order to rest at the appropriate time. Consequently, humans feel and perform differently from one time of day to another. These changes are called psychological circadian rhythms and are driven by the endogenous circadian pacemaker [28].

The circadian rhythm, inherent to all human beings, consists in 24 hours of biological patterns that affects the fatigue level of a person [29]. All the rhythms develop vital tasks of bio-regulation allowing the body to maintain normal levels of motivation, effort, mental, and physical health [30]. When these rhythms are disrupted, performance is degraded and health can be affected. The circadian rhythm varies in a period of 24 hours while other rhythms may vary over a period of weeks, months or years. The main function of the circadian rhythm is to prepare the person to sleep through the hormonal regulation of the most basic functions of the human body [31].

Spencer [27] conducted an experiment to examine the variation of human performance throughout the day due to the influence of the circadian rhythm. Based on the results of this experiment, the author developed an equation that relates the time of day with the amount of time a person is awake until that moment to predict the performance of operators at different times of day. Therefore, this author observed that the average productivity percentage in performing a task is represented by the Digit Symbol Substitution Task (DSST), where T and $t$ are measured in hours and $T$ is the time of day and $t$ the time elapsed from awakening until moment $T$.

$$
\operatorname{DSST}(T, t)=233,3+1,54 t-0,304 t^{2}+0,0108 t^{3}+4,97 \cos (2 \pi(T-17,05) / 24)
$$

\section{MODEL DEVELOPMENT}

Simulation should be used when you want to predict the effect of changes in the system or evaluate its performance or behaviour. It is used in actual problem solving, during operations management, which involves processes of design, planning, control and operation, whether in manufacturing or services industries [32].

The simulated systems are processes that belong to different companies and were chosen because they are production lines where manual work is predominant, since this type of activity is greatly influenced by the human factor.

These systems were carefully chosen since they presented important features essential for the desired analysis, such as the presence of intensive manual labour in all activities. This aspect is particularly important because in these cases, the human factor has a direct influence on the performance of the system and the circadian rhythm becomes more significant. Another important point is that the presence of tasks with low cycle time in the systems causes the operators to be more prone to fatigue and performance variation; measuring this variation is the goal of the circadian rhythm equation used in the agent-based simulation models. It is worth remembering that we are dealing with stochastic systems where the analyses are by no means trivial. 


\section{System 1}

The first system to be simulated is a manufacturing cell of a high-tech company. The product manufactured in this cell represents about $40 \%$ of the company revenues. The cell operates a single shift from 8:00 am to 5:00 pm, with a one-hour lunch break and 30-minute coffee break, distributed at intervals along the shift. There are two operators available to assemble the product in question and the activities undertaken by these operators are manual. In Fig. 1, we have the conceptual model of the productive process of the system constructed using the IDEF-SIM modelling technique [33].

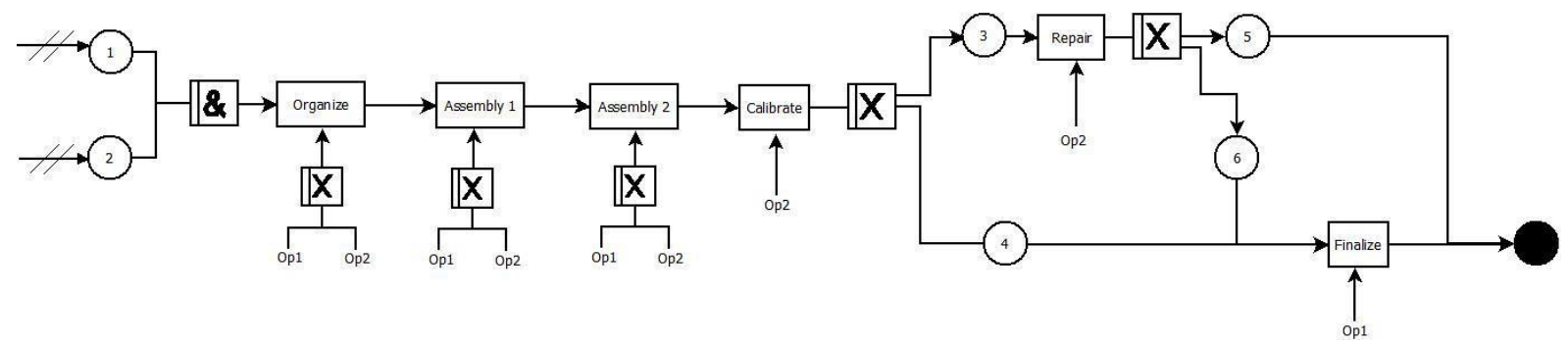

Figure 1: Conceptual model of the system 1.

\section{System 2}

The second system to be simulated is a section of a wiring harness production line, which is responsible for producing kits that are routed in the assembly table of these wiring harnesses. Each cell operator inserts a pre-set number of cables and delivers their assembly to the subsequent cell through rails, known as kit rail. The operator of the last cell delivers the final Kit to be routed in the conveyor tables. Note that the conceptual model of the production process shown in Fig. 2 was built by using the IDEF-SIM technique. The work hours are between 8:00 am and 5:00 pm, with a break between 12:00 and 1:00, when the workers are absent from the production line.

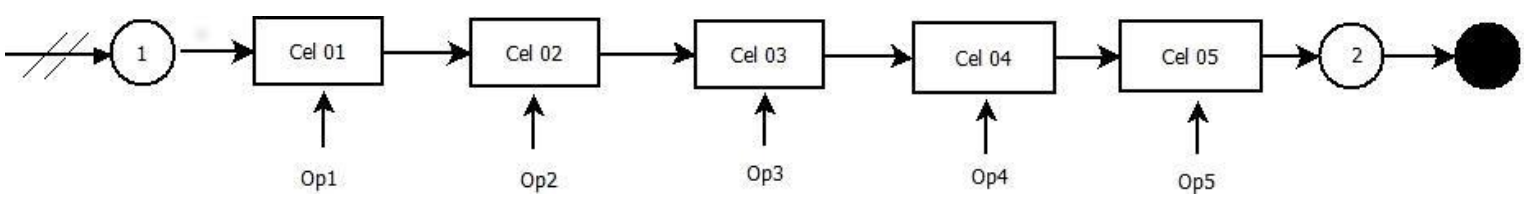

Figure 2: Conceptual model of the system 2.

For the application of discrete-event simulation (DES) and agent-based simulation (ABS), it is required modelling the system under study in a computer system to accelerate the execution of activities. The software used was the Anylogic $\AA$, since it allows the construction of models with the integration of agent-based simulation and discrete-event simulation. The purpose of building these models is to represent the human element through agents susceptible to factors that affect human performance; in this case the circadian rhythm was chosen in order to better represent the human element and its behaviour. It is worth noting that each employee is represented by an agent who can decide what time to wake up, which is an important variable in calculating the circadian rhythm equation.

The main entry data for the model development is the lead time for manual activities of each worker in its respective workstation. Usually, in simulation projects, the data inputted in the computational model comes from time measurement and their average values, or the probability distribution values. However, it is important to point out that the majority of the DES articles used on the systematic review had timing measurement as their way to represent the variation of human performance. Nevertheless, in many occasions it is necessary a significant number of collected data to find the best probability distribution. 
One of the main weaknesses of operating DES is the exertion needed and costs spent on collecting and handling the input data from different organisation's data resources [20]. In this research, for the hybrid simulation model, the time variation of the operators, both for increase and for reduction of the task time, will be calculated by the circadian rhythm equation, therefore, its required only the average of ten timing measurement.

In the computer models built, employees are represented by agents who are affected by the circadian rhythm effect throughout the day. As presented in the literature review, the circadian rhythm directly affects human beings, their performance and consequently their productivity. According to the equation of [27], performance variation should be calculated at each hour considering the variables "time the person wakes up" and "difference between the time in consideration and the time the person wakes up".

For the computer model, at every day hour, the performance variation of each agent is calculated. This variation is multiplied by the duration of the activity, penalizing or adding this time according to the result of the equation. Note that the time used in the models for each activity was the average time of each activity, as provided by the companies.

With respect to agents' decision making concerning the time to wake up, there is a parameter in the models that represents the time a worker will awaken, variable used in Eq. (1). The value of this variable is a discrete uniform distribution between 5 and $7 \mathrm{am}$. The choice of these values is justified by the time that employees of the cell usually wake up.

Fig. 3 shows a diagram representing how agents provide the data necessary to calculate performance and how they are affected by the result of this equation, at every hour of the workday.

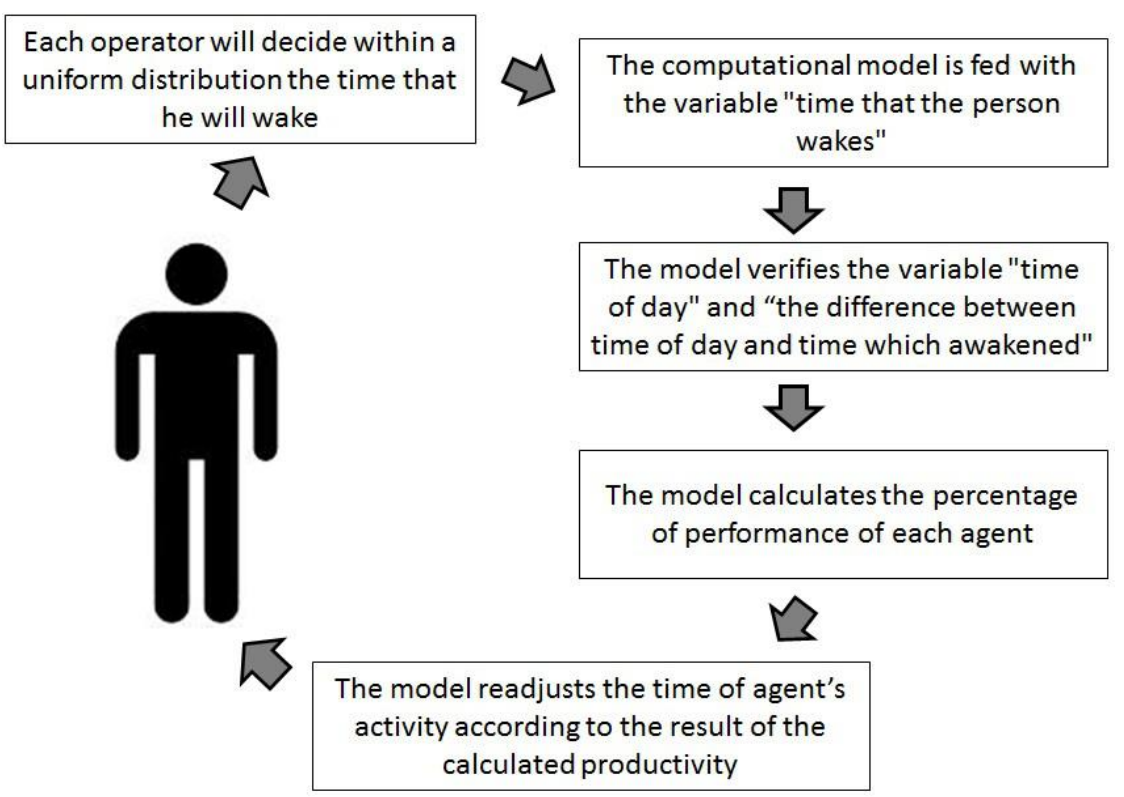

Figure 3: Diagram considered for representing of each agent in both systems.

The agent performance for both systems models is represented in Fig. 4. Each operator agent, when initialized, has the value of its parameter "wakeUpTime" randomly generated within a uniform distribution and the value selected will represent the time that the operator will awaken. Then the agent enters the state "Working," which is the time he will be working. While in this state, a transition called "updateProductivity" performs an hourly update on the operator productivity using the circadian rhythm equation, thus changing the variable "productivity". This variable will directly affect the execution time of the tasks, reducing or increasing this time according to the calculated hourly productivity. 


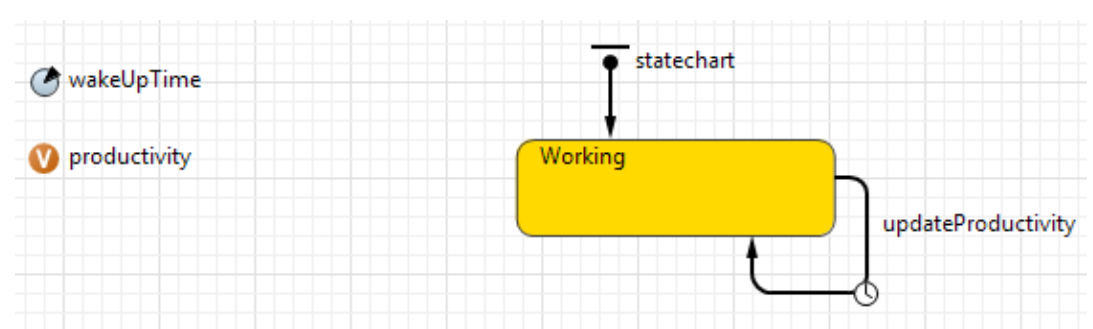

Figure 4: Agent's behaviour in the built models.

\section{MODEL VALIDATION}

The main goal of the computer models built was, by combining discrete-event simulation with agent-based simulation, to better represent the human element in the simulation of production processes, considering their autonomy in decision-making and performance affected by the circadian rhythm. Thus, it became necessary to validate these computer models developed to ensure that they represent the actual system behaviour.

One way to validate the computer model is through historical data validation and if historical data exists, some of these data can be used to build the model and the remainder used to determine if the model behaves like the system [34]. The output variable "monthlyproduced parts" was considered for the models validation.

The data generated by the computer models related to productivity were compared to actual data collected from the systems. As the number of data collected in the actual systems is small, relating to the time of follow-up of the cell, there is no sufficient basis to state that the data represents a normal distribution.

This fact does not affect the statistical analysis, since when the distribution study is not characterized as normal, non-parametric methods such as the Mann-Whitney $U$ test can be used [35]. Also according to the authors, this test compares two independent samples, where the null hypothesis is tested to verify that the means of the two samples are equal. Now the alternative hypothesis is related to the inequality of the means. If the $p$-value is not less than 0.05 , it is possible to conclude that there is insufficient evidence to reject H0. Therefore, the data does not support the hypothesis that there is a difference between the population medians.

So when performing the Mann-Whitney $U$ nonparametric test for the data of the study, we can say with $95 \%$ confidence that the two samples of both models, actual and simulated, are considered statistically equal, that is, we can consider that the computer model built represents the behaviour of the cell in question. Table I shows the results of statistical test of the two models.

Table I: Mann-Whitney $U$ Test results performed in Minitab ${ }^{\circledR}$.

\begin{tabular}{|l|c|c|}
\cline { 2 - 3 } \multicolumn{1}{c|}{} & System 1 & System 2 \\
\hline Real Median & 101.5 & 184 \\
\hline Real Simulated & 143.5 & 182 \\
\hline$P$-value & 0.3785 & 0.1020 \\
\hline
\end{tabular}

\section{ANALYSIS OF THE RESULTS}

Some authors support the idea that discrete-event simulation overestimates the production capacity of simulated systems by not correctly representing the human factor. Therefore, in order to make comparisons between the simulation types, a discrete-event simulation model was constructed for each system. In these models, the execution time of operators activities is 
related to the distribution of probabilities found for the collected times in actual timing of the line.

In order to exemplify how the time data were used in the construction of the discrete events simulation models, Table II shows the probability distribution found in the workstation of System 2. It is important to remember that the same procedure was used in System 1.

It is important to say that the time required for time collection in both systems was approximately two months. It is also important to point out that the same procedure of the validation statistical test was performed for the results of the discrete-event simulation model and it shows that this model is also validated, since the average of actual and simulated samples are statistically equal.

Table II: Time probability distribution for System 2 cells.

\begin{tabular}{|c|c|c|c|}
\hline Cell & Probability Distribution & Cell & Probability Distribution \\
\hline Cell 01 & Beta $(122,156,1.47,1.36) \mathrm{sec}$ & Cell 04 & Lognormal $(126,2.69,0.431) \mathrm{sec}$ \\
\hline Cell 02 & Beta $(121,161,2.69,2.82) \mathrm{sec}$ & Cell 05 & Beta $(124,155,1.96,2.21) \mathrm{sec}$ \\
\hline Cell 03 & Loglogistic $(130,3.5,13.5) \mathrm{sec}$ & & \\
\hline
\end{tabular}

Productivity results of DES and ABS combined with DES models were compared to the actual productivity results collected from the system. Thus, it is possible to check the behaviour of the two types of simulation compared with the actual system. It's worth recalling that in the DES model resources represent the human element where activity time is related to a probability distribution derived from timing. However, in the ABS combined with DES model, operators are represented by agents, where the task execution time is affected by the circadian rhythm.

In the graph of Fig. 5, we have a comparison of the monthly productivity result of the simulation models with the actual productivity of the system. It can be observed through the graph that the actual productivity of this system presents a highly variable behaviour. One of the reasons for this variation may be the fact that it is a newly built production cell, where operators do not yet have the experience required to manufacture the product analysed. The actual productivity monitoring time is limited to the value of six months also by the fact that it is a line in the beginning of production and does not have historical data. Despite this troubled behaviour of the cell, the ABS is closer to the actual behaviour curve of the system.

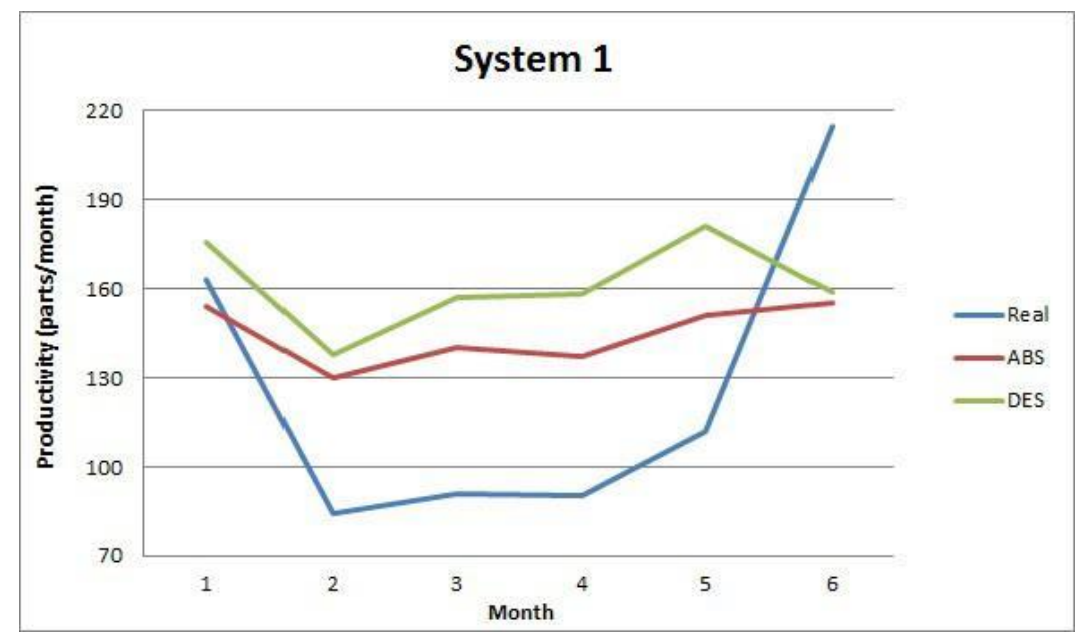

Figure 5: Results of computer models of the real system 1.

On the other hand, the graph of Fig. 6 shows the results of daily productivity of the DES and ABS models compared to the results collected from the actual system 2. Data from the 21 
days of monitoring the production line was considered to create the graph, this being the time concerning to a month of production. The actual system behaviour is not as variable as the previous system because in the system under consideration, operators have extensive experience assembling the product. Thus, the production time does not vary much and consequently the daily production also does not vary widely. It is worth mentioning that on the $14^{\text {th }}$, the daily production suffered a relative decline because it was a day of audit in the company and the time available for production was reduced, affecting productivity. This reduction of production time was passed on to computer models to the best represent reality. Through the graphic results it can be seen that while both models present behaviours similar to the actual system, the ABS combined with DES model is closer to the actual system productivity values.

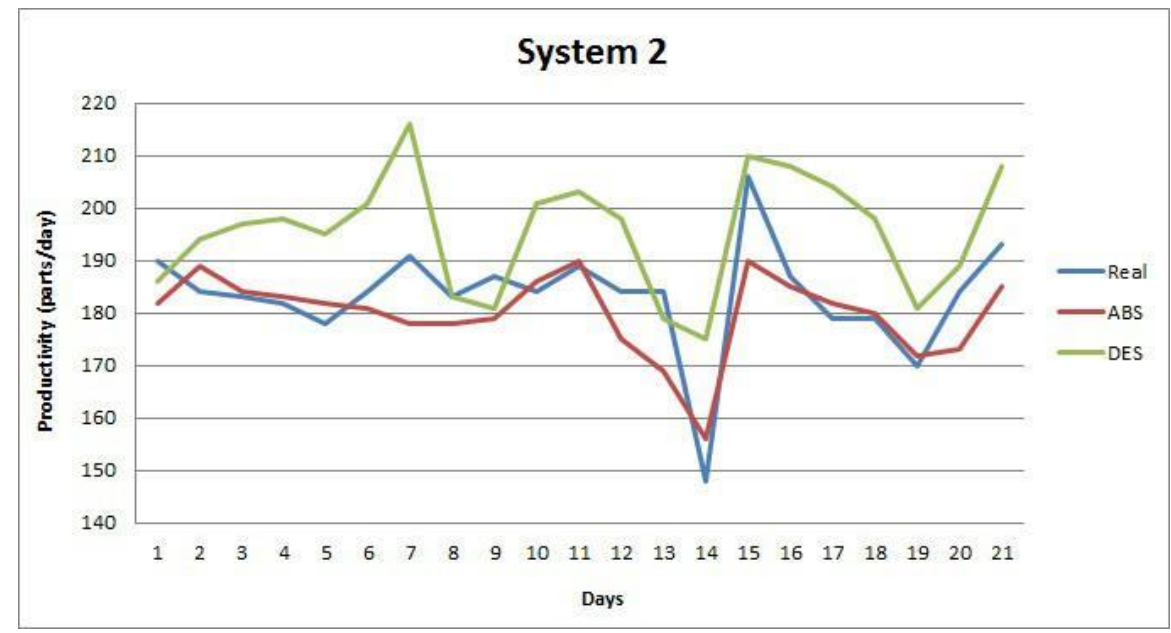

Figure 6: Results of computer models of the real system 2.

It can be seen from the graphs that the results of the computer models that integrate ABS with DES with insertion of the circadian rhythm tend better to the actual system behaviour than models of discrete-event simulation. In order to quantify this difference, Table III was developed by comparing the averages of relative errors, that is to say the average of the percentage differences between the simulated and the actual result for each data collected. The average of relative errors is smaller for ABS models combined to DES in both cases.

Table III: Average relative errors.

\begin{tabular}{|c|c|c|c|c|}
\cline { 2 - 5 } \multicolumn{1}{c|}{} & \multicolumn{2}{c|}{ System 1 } & \multicolumn{2}{c|}{ System 2 } \\
\cline { 2 - 5 } \multicolumn{1}{c|}{} & DES Model & ABS+DES Model & DES Model & ABS+DES Model \\
\hline Average Relative Error & $51.3 \%$ & $38.2 \%$ & $7.6 \%$ & $3.6 \%$ \\
\hline
\end{tabular}

However, it can be observed that in System 1, the average relative errors of the models compared to the Actual system is considerably large. That is due to the fact that this system has a highly variable behaviour since it is a system that recently began assembling the product in question. Operators of this cell still have little ability to produce the item, presenting variation in assembling times and consequently in productivity. The difference between the simulated and the actual results for System 1 is relatively large, but the ABS model presents a smaller error when compared to DES, which better represents the actual system analysed.

One last comparison was held, now only between the simulation models. In this new analysis, the productivity results were compared between the DES and ABS/DES computer models to determine whether these models have the same results. After conducting a 
hypothesis test between the samples of simulated results, we can say with $95 \%$ confidence that the samples are considered statistically the same for both systems, i.e. the results of the DES and ABS/DES models are statistically equal.

It is important to mention that in discrete-event simulation projects, when it is not intended to represent the human element activity with deterministic times, a large collection of time data is required in order to find a statistical distribution that comes close to the activities actual time. These distributions are used to feed the computer model and therefore, the closer they are to actual times, the higher the probability of this model to be statistically validated. It was necessary collecting 200 time data for the system 1 and 500 time data for system 2 to find the best probability distribution of time.

However, only the average of 10 time data of activities plus the change in the circadian rhythm for each agent were used for the models presented in this paper, and the result of this model was statically validated. This represents an advantage, since an intense timing, which generally requires a large data collection; period to find the best statistical distribution was not required for the construction of this model.

\section{CONCLUSION}

According to some authors, discrete-event simulation alone does not properly represent the human factor and the features that affect its productivity because it represents the human element as simple resources. Now, agent-based simulation makes it possible to represent the human resource as autonomous, intelligent and proactive agents, which are characteristics closely related to the behaviour of the human element.

Thus, two models of different production processes were built combining the ABS with DES in order to verify that agent-based simulation is a possible solution for the existent gap in the human factor representation in discrete-event simulation, where the human factor has a direct impact in the process performance.

The operators were represented in the model as agents, so it was possible to insert one of the features that affect human performance, in this case, the circadian rhythm.

Agents, by nature, are decision makers, and in these models each agent decides within a probability distribution what time he would wake up, giving each user a different biorhythm, making possible the stochastic analysis of the effect of the circadian rhythm on productivity.

A nonparametric hypothesis testing was conducted to verify that the data generated in the computer models are statistically equal to the actual data collected. It was verified that in both cases analysed the samples are statistically equal, i.e., the computer model that integrates the ABS and DES represents the behaviour of the cell in question. It is worth noting that intense timing of the activities time data was not necessary, since it was possible to validate the computer model with the average of these times and the circadian rhythm.

A comparison between the model built combining ABS, DES and circadian rhythm and a DES model where simple resources represent operators and the time of their activities is a probability distribution was also performed for each system. It was verified, for the studied systems, that the ABS model integrated to DES represents better the actual system behaviour than the discrete-event simulation model, showing a lower average of relative error. Besides, it was not necessary an intense timing for the ABS model, which generally requires a large data collection period to find the best statistical distribution.

This article demonstrates how agent based simulation can be added to the discrete-event simulation to represent the human factor in production systems. This hybrid simulation allowed simulating the human element influenced by a feature that affects the productivity, the circadian rhythm. It is suggested that this structure is used to represent the human element in simulation projects with intensive manual labour, especially in situations where the system 
is new, with no historical data available, or when it is not easy or too time consuming to collect and analyse such data.

Future work may include the selection of other factors that might affect human productivity, such as age of the employees, ambient temperature, among others, to verify whether these factors help in a better representation of the human element in simulation.

\section{ACKNOWLEDGEMENTS}

The authors would like to thank the development agencies Capes, Fapemig and CNPq.

\section{REFERENCES}

[1] Negahban, A.; Yilmaz L. (2014). Agent-based simulation applications in marketing research: an integrated review, Journal of Simulation, Vol. 8, No. 2, 129-142, doi:10.1057/jos.2013.21

[2] Ahmed, R.; Shah, M.; Umar, M. (2016). Concepts of simulation model size and complexity, International Journal of Simulation Modelling, Vol. 15, No. 2, 213-222, doi:10.2507/ IJSIMM15(2)2.317

[3] O'Kane, J. F.; Spenceley, J. R.; Taylor, R. (2000). Simulation as an essential tool for advanced manufacturing technology problems, Journal of Materials Processing Technology, Vol. 107, No. 1-3, 412-424, doi:10.1016/S0924-0136(00)00689-0

[4] Borshchev, A. (2013). The Big Book of Simulation Modeling, AnyLogic North America, Chicago

[5] Dubiel, B.; Tsimhoni, O. (2005). Integrating agent based modeling into a discrete event simulation, Proceedings of the 2005 Winter Simulation Conference, 1029-1037

[6] Baines, T.; Mason, S.; Siebers, P.-O.; Ladbrook, J. (2004). Humans: the missing link in manufacturing simulation?, Simulation Modelling Practice and Theory, Vol. 12, No. 7-8, 515526, doi:10.1016/S1569-190X(03)00094-7

[7] Siebers, P.-O. (2006). Worker performance modeling in manufacturing systems simulation: Proposal for an agent-based approach, Rennard, J.-P. (Ed.), Handbook of Research on Nature Inspired Computing for Economics and Management, Idea Group Inc., Hershey, 661-678

[8] Chan, W. K. V.; Son, Y. J.; Macal, C. M. (2010). Agent-based simulation tutorial - simulation of emergent behavior and differences between agent-based simulation and discrete-event simulation, Proceedings of the 2010 Winter Simulation Conference, 135-150

[9] Siebers, P. O.; Macal, C. M.; Garnett, J.; Buxton, D.; Pidd, M. (2010). Discrete-event simulation is dead, long live agent-based simulation!, Journal of Simulation, Vol. 4, No. 3, 204-210, doi: $10.1057 /$ jos.2010.14

[10] Banks, J. (1999). Introduction to simulation, Proceedings of the 1999 Winter Simulation Conference, 7-13

[11] Harrell, C.; Ghosh, B. K.; Bowden, R. (2000). Simulation Using Promodel, $3^{\text {rd }}$ ed., McGraw-Hill, Boston

[12] Giannasi, F.; Lovett, P.; Godwin, A. N. (2001). Enhancing confidence in discrete event simulations, Computers in Industry, Vol. 44, No. 2, 141-157, doi:10.1016/S0166-3615(00)000841

[13] Baines, T. S.; Asch, R.; Hadfield, L.; Mason, J. P.; Fletcher, S.; Kay, J. M. (2005). Towards a theoretical framework for human performance modelling within manufacturing systems design, Simulation Modelling Practice and Theory, Vol. 13, No. 6, 486-504, doi:10.1016/ j.simpat.2005.01.003

[14] Macal, C. M.; North, M. J. (2009). Agent-based modeling and simulation, Proceedings of the 2009 Winter Simulation Conference, 86-98

[15] Leitão, P. (2009). Agent-based distributed manufacturing control: A state-of-the-art survey, Engineering Applications of Artificial Intelligence, Vol. 22, No. 7, 979-991, doi:10.1016/ j.engappai.2008.09.005

[16] Zhang, H. P. (2015). An agent-based simulation model for supply chain collaborative technological innovation diffusion, International Journal of Simulation Modelling, Vol. 14, No. 2, 313-324, doi:10.2507/IJSIMM14(2)CO6 
[17] Siebers, P.-O.; Onggo, S. (2014). Graphical representation of agent-based models in Operational Research and Management Science using UML, Proceedings of the $7^{\text {th }}$ Operation Research Society Simulation Workshop, 143-153

[18] Joo, J.; Kim, N.; Wysk, R.A.; Rothrock, L.; Son, Y.-J.; Oh, Y.; Lee, S. (2013). Agent-based simulation of affordance-based human behaviors in emergency evacuation, Simulation Modelling Practice and Theory, Vol. 32, 99-115, doi:10.1016/j.simpat.2012.12.007

[19] Hughes, K.; Jiang, X. (2010). Using discrete event simulation to model excavator operator performance, Human Factors and Ergonomics in Manufacturing \& Service Industries, Vol. 20, No. 5, 408-423, doi:10.1002/hfm.20191

[20] Barlas, P.; Heavey, C.; Dagkakis, G. (2015). An open source tool for automated input data in simulation, International Journal of Simulation Modelling, Vol. 14, No. 4, 596-608, doi:10.2507/IJSIMM14(4)3.306

[21] Zhao, C.; Zhang, X.; Qiu, J. (2012). Modelling and simulation on collaborative work in cellular manufacturing, Proceedings of the International Conference on Computer Supported Cooperative Work in Design, 730-733

[22] Baines, T. S.; Hadfield, L.; Mason, S.; Ladbrook, J. (2003). Using empirical evidence of variations in worker performance to extend the capabilities of discrete event simulations in manufacturing, Proceedings of the 2003 Winter Simulation Conference, Vol. 2, 1210-1216

[23] Baines, T. S.; Kay, J. M. (2002). Human performance modelling as an aid in the process of manufacturing system design: a pilot study, International Journal of Production Research, Vol. 40, No. 10, 2321-2334, doi:10.1080/00207540210128198

[24] Kasaie, P.; Kelton, W. D. (2015). Guidelines for design and analysis in agent-based simulation studies, Proceedings of the 2015 Winter Simulation Conference, 183-193

[25] Lazarova-Molnar, S. (2013). Agent-based modeling and simulation framework for enhanced project schedules, $20^{\text {th }}$ International Congress on Modelling and Simulation, 887-893

[26] Kavicka, A.; Klima, V.; Adamko, N. (2007). Simulations of transportation logistic systems utilising agent-based architecture, International Journal of Simulation Modelling, Vol. 6, No. 1, 13-24, doi:10.2507/IJSIMM06(1)2.075

[27] Spencer, M. B. (1987). The influence of irregularity of rest and activity on performance: a model based on time since sleep and time of day, Ergonomics, Vol. 30, No. 9, 1275-1286, doi: $10.1080 / 00140138708966022$

[28] Monk, T.; Buysse, D.; Reynolds III, C.; Berga, S.; Jarret, D.; Begley, A.; Kupfer, D. (1997). Circadian rhythms in human performance and mood under constant conditions, Journal of Sleep Research, Vol. 6, No. 1, 9-18, doi:10.1046/j.1365-2869.1997.00023.x

[29] Zhang, H.; Yan, X.; Wu, C.; Qiu, T. (2014). Effect of circadian rhythms and driving duration on fatigue level and driving performance of professional drivers, Transportation Research Record: Journal of the Transportation Research Board, Vol. 2402, 19-27, doi:10.3141/2402-03

[30] Marquie, J. C.; Foret, J. (1999). Sleep, age, and shiftwork experience, Journal of Sleep Research, Vol. 8, No. 4, 297-304, doi:10.1046/j.1365-2869.1999.00170.x

[31] Monk, T. H.; Leng, V. C. (1982). Time of day effects in simple repetitive tasks: some possible mechanisms, Acta Psychologica, Vol. 51, No. 3, 207-221, doi:10.1016/0001-6918(82)90035-X

[32] Bertrand, J. W. M.; Fransoo, J. C. (2002). Operations management research methodologies using quantitative modeling, International Journal of Operations \& Production Management, Vol. 22, No. 2, 241-264, doi:10.1108/01443570210414338

[33] Montevechi, J. A. B.; Leal, F.; Pinho, A. F.; Costa, R. F. S.; Oliveira, M. L. M.; Silva, A. L. F. (2010). Conceptual modeling in simulation projects by mean adapted IDEF: An application in a Brazilian tech company, Proceedings of the 2010 Winter Simulation Conference, 1624-1635

[34] Sargent, R. G. (2013). Verification and validation of simulation models, Journal of Simulation, Vol. 7, No. 1, 12-24, doi:10.1057/jos.2012.20

[35] Montgomery, D. C.; Runger, G. C. (2003). Applied Statistics and Probability for Engineers, $3^{\text {rd }}$ ed., John Wiley \& Sons, New York 